\title{
LA DIRECTIVA DE PRODUCTOS DE CONSTRUCCIÓN La normalización en Europa y España
}

Gonzalo Sotorrío, Responsable del Área de Construcción. División de Normalización - AENOR. ESPAÑA.

$769-12$ 


\section{NOTA:}

En diferentes ocasiones, la Revista INFORMES DE LA CONSTRUCCIÓN ha abierto sus páginas a colaboraciones y textos normativos sobre el importante tema de la normalización de productos de construcción. Una vez más, ofrece en CUADERNOS un trabajo que, aun sienđo de autor (lo cual no es usual en esta Sección), presenta un indudable interés divulgador del estado de la cuestión. 


\section{LA DIRECTIVA DE PRODUCTOS DE CONSTRUCCIÓN La normalización en Europa y España}

\section{O. INTRODUCCIÓN}

Existen varias directivas que afectan a esta industria tan diversa de la construccion y podemos citar entre otras la relacionada con las "compras públicas", sin contar con todas aquellas que recogen temas fiscales y financieros; pero la Directiva de Productos de Construcción 89/106, en cuanto al aspecto técnico de cualquier obra constructiva, es la piedra angular para la consecución de ese mercado único y transparente que la Comunidad Europea se ha impuesto como un objetivo de indudables ventajas políticas y económicas para sus países miembros.

El desarrollo económico de cualquier comunidad se basa en gran medida en unas infraestructuras importantes y en un sector de construcción vivo y dinámico y la Comunidad Europea no puede ser ajena a estas premisas que han demostrado su validez en numerosos paises.

\section{LAS NORMAS EN LA DIRECTIVA}

La referencia más clara a las normas está contenida en el capítulo II, artículo 7 que se refiere a las "Normas armonizadas", especificándose que los organismos europeos de normalización deberán establecer las normas armonizadas de acuerdo con mandatos conferidos por la Comisión de la Comunidad, debidamente asesorada por el Comité Permanente de la Directiva y de conformidad con los procedimientos que fija la Directiva 83/189/CEE.

También se recoge que las referencias de estas normas, una vez establecidas, se publicarán en la serie "C" del Diario oficial de lás Comunidades Europeas, el conocido DOCE.

En el artículo 12 se indica que dichas normas armonizadas se elaborarán tomando como base los "documentos interpretativos" que dan forma concreta a cada uno de los seis requisitos esenciales que establece la Directiva.

Estos mandatos definitivos de normalización, no provisionales como hasta ahora, no se han producido, pues requieren para su redacción los documentos interpretativos que están concluyéndose en estos momentos. Es decir que las normas armonizadas con los requisitos que anteriormente indicamos aún no se han elaborado.

Para estos casos el artículo 20 establece como una de las misiones del Comité Permanente el reconocimiento de las especificaciones nacionales que se consideran conformes con los requisitos esenciales. Este es un punto de indudable importancia, pues las normas europeas que actualmente se están elaborando, serán normas nacionales iguales a los 18 países miembros del Comité Europeo de Normalización (CEN) en virtud de las reglas comunes que nos obligan a todos los países miembros. 
Resulta por ello evidente, la importancia que tiene el participar activamente en la elaboración de las normas actualmente en curso en CEN ya que se está estableciendo un conjunto de especificaciones técnicas que van a ser comunes a todos los países europeos y que entendemos pueden constituir las reglas técnicas que permitan la puesta en vigor de la Directiva.

\section{DOCUMENTOS INTERPRETATIVOS Y MANDATOS}

Como ya se indica anteriormente los documentos interpretativos y los mandatos son piezas esenciales para llegar a contar con unas especificaciones técnicas armonizadas que son en realidad los documentos que van a hacer posible la total y completa puesta en vigor de la Directiva para cada producto de construcción.

La Directiva afirma que los productos destinados a ser incorporados en las obras, tanto de edificación como de ingeniería civil, deberán cumplir los requisitos esenciales que se establecen, durante un período de vida económicamente razonable.

Dichos requisitos esenciales son seis:

- Resistencia mecánica y estabilidad.

- Seguridad en caso de incendio.

- Higiene, salud y medio ambiente.

- Seguridad de utilización.

- Protección contra el ruido.

- Ahorro de energía y aislamiento térmico.

Estos requisitos esenciales son declaraciones tan generales y recogen unos conceptos tan poco concretos que exigen, para ser operativos, una concreción. Esta necesidad ha sido prevista por los propios legisladores, de modo que se ha previsto que cada uno de ellos sea plasmado en un "documento interpretativo". Estos documentos constituyen la concreción de cada uno de esos requisitos y deben, además, armonizar las terminologias y las bases o principios técnicos de cada requisito llegando incluso a fijar distintos niveles de exigencias, si asi se considera necesario.

La preparación de estos documentos corresponde a la Comisión de la CE, sirviéndose para ello del Comité Permanente de la Directiva, que constituido por representantes de las Administraciones de los doce miembros comunitarios es pieza esencial y especializada que debe evacuar todas las dudas y aprobar todos los aspectos que hagan posible la puesta en vigor de la Directiva. La redacción de dichos documentos interpretativos está siendo llevada a cabo por Comités Técnicos, tantos como requisitos esenciales, y que formados por delegaciones designadas por las Administraciones de los países miembros están a punto de finalizar su trabajo. Pronto se podrá contar con dichos documentos y conocer cuáles y cómo son los aspectos a tener en cuenta para cumplir cada requisito esencial.

Una de las misiones más destacadas, si no la principal, que la Directiva reserva para estos documentos es que sirvan de referencia para la elaboración de las especificaciones técnicas que forman la base de la Directiva. Es decir, las normas armonizadas y los documentos de idoneidad técnica europeos (DITE).

Los mandatos de normalización a que antes nos referiamos serán preparados por la Comisión y constituirán el encargo o "contrato" que ésta establecerá con el Comité Europeo de Normalización (CEN) para que éste elabore las normas armonizadas.

Se prevé que la forma en que el Comité Permanente va a preparar los mandatos para que la Comisión los lleve a efecto será: para cada producto de construcción se estudiará qué requisito o requisitos esenciales le afectan y por tanto el órgano de trabajo de CEN a quién se encomiende el trabajo, contará para elaborar la norma con los documentos interpretativos que corresponden a cada requisito esencial que 
afecta al producto. Sabrá así qué aspectos debe especificar, si debe fijarse niveles para alguno de ellos y constituirá en definitiva una guía para fijar cuál debe ser el contenido de la norma.

Este proceso a que nos hemos referido servirá igualmente para elaborar de manera similar los DITES o serán simples elementos de trabajo esenciales para adoptar decisiones que el Comité Permanente de Construcción tenga que tomar sobre cualquier cuestión de reconocimiento de normas nacionales 0 similares.

\section{PANORAMA DE LA NORMALIZACIÓN EUROPEA EN EL SECTOR CONSTRUCCIÓN}

Si bien resulta siempre comprometido establecer fronteras en este sector dada la gran cantidad de equipos, elementos y productos que entran a formar parte de cualquier obra constructiva y máxime ahora que los edificios van ganando complejidad por lo que contienen, necesitamos, con el fin de proceder a un estudio de la situación, fijar unas fronteras y adoptar decisiones de qué incluimos en el sector y qué no, reconociendo la subjetividad en muchos casos de esta decisión.

Viene en nuestra ayuda para establecer esas fronteras el propio CEN con su clasificación. Tendiendo a ella, podemos considerar que hay actualmente 35 Comités Técnicos (véase anexo 1) realizando trabajos de normalización que van desde las especificaciones de cementos o componentes del hormigón hasta elementos constructivos más complejos como pueden ser los herrajes y cerraduras o los equipos de climatización.

Todos estos Comités Técnicos se estructuran en subcomités o directamente en Grupos de Trabajo o Grupos especializados (TG), creándose una estructura con numerosas ramificaciones de forma que en este momento hay un total de 395 órganos de trabajo funcionando en CEN dentro de este sector.

Resulta interesante precisar dentro de esta oganización los matices que diferencian unos órganos de trabajo de otros. En los Comités y Subcomités las delegaciones de los países participantes acuden, acreditados por cada organismo de normalización nacional, con su carácter de representantes nacionales, es decir sus opiniones y las posturas que mantengan tienen que responder a la opinión consensuada de los industriales, consumidores, Administración, centros de investigación y profesionales de su país. Resulta por tanto fundamental la discusión y la preparación de dichas posturas en reuniones de todas las partes implicadas. Es decir, en definitiva, que deben adoptarse en reuniones de los Comités nacionales que son el punto de encuentro de todos ellos.

Por el contrario, los participantes en los Grupos de Trabajo (WG) y Grupos especializados (TG) es en condición de expertos, nominados por su país, pero a título personal; si bien resulta lógico que informen adecuadamente y recaben las opiniones a mantener, en las reuniones a los Comités Nacionales que en su día propusieron su designación al organismo nacional de normalización que es el que reglamentariamente acredita.

Otro dato de interés son los problemas de solape que se originan y la necesidad de perfecta conjunción y coordinación de trabajos que debe existir entre los Comités Técnicos de CEN que normalizan productos y los que fijan características exigibles para cubrir un servicio, independientemente de los productos que las ofrecen. Nos estamos refiriendo a que la coordinación en muchos casos es difícil entre los Comités Técnicos de producto y los de uso, como habitualmente se les denomina.

\subsection{El Consejo Técnico Sectorial de la Construcción}

Dentro del funcionamiento del CEN se han creado recientemente los Consejos Técnicos Sectoriales, conocidos oficialmente como CEN/BTS. 
Se ha tratado con esto de descargar de trabajo y especializar la toma de decisiones en tres áreas muy determinadas: construcción, ingeniería y sanidad.

Las misiones encomendadas a estos Consejos sectoriales, dentro de su campo de actividad especializado y"referido a los Comités Técnicos que se le han asignado, son entre otros los siguientes:

a) Coờdinar y planificar los trabajos de normalización en su área.

b) Controlar la marcha de los trabajos en curso y vigilar el cumplimiento de los plazos establecidos para cada norma europea.

c) Revisar los títulos, campos de actividad y programas de trabajo de los Comités Técnicos que le han sido asignados.

d) Evaluar los resultados de las encuestas sobre nuevos trabajos.

e) Decidir sore los PQ (Cuestionarios preliminares) que deben emitirse y evaluar los resultados.

f) Asignar nuevos trabajos a los Comités que dependen de él.

g) Recomendar al Consejo Técnico (CEN/BT) la creación de nuevos Comités y la asignación de las Secretarías de los mismos.

h) Aprobar el nombramiento de Presidentes de Comités Técnicos y la creación de subcomités.

i) Autorizar el establecimiento de coordinaciones con organizaciones técnicas, científicas o profesionales, europeas.

Estas son algunas de las más destacadas tareas encomendadas a los BTS aparte de aquellas otras que expresamente les delegue el Consejo Técnico del CEN.

Dentro de esta nueva orientación que ha emprendido CEN, el BTS 1 es el de "Construcción e Ingenieria Civil" cuyas misiones son hacer el seguimiento y control de todos los trabajos de normalización que se derivan de la puesta en práctica de la Directiva 89/106, es decir, la de Productos de Construcción que estamos tratando aquí.

El precedente de este Consejo Técnico para la construcción es el antiguo CEN/PC 1 o Comité de Programación de larga tradición y que venia desarrollando hasta el año pasado las labores de control y planificación en el campo de la construcción y que ahora se han visto potenciadas con esta nueva organización.

\subsection{Los Eurocódigos se traspasan a CEN}

Otra novedad que consideramos de interés destacar es que los conocidos Eurocódigos, es decir los documentos que establecen reglas de diseño y ejecución a nivel europeo, se acaban de traspasar al ámbito del CEN, es decir van a ser normas europeas en un plazo más o menos corto. Todo ello gracias al acuerdo a que han llegado la Comisión de las Comunidades y el CEN.

Conviene resaltar que el carácter singular de estos documentos ha hecho imprescindible llegar a ciertas excepciones que hacen que las reglas internas de tipo general que tiene establecidas CEN, se adapten a este caso singular. De cualquier manera el hecho importante para el mundo de la construcción es que los Eurocódigos van a ser normas europeas con todo lo que ello significa de amplia difusión y concurso de todos los interesados durante su elaboración, asi como documentos comunes de referencia para todos los países, cuando estén elaborados.

Para llevar a cabo los trabajos se ha constituido un Comité Técnico específico, el CEN/TC 250 y que ha acordado crear tantos subcomités como Eurocódigos van a elaborarse, es decir, ocho. 
Dentro de las.singularidades a que haciamos referencia anteriormente destacaremos que en las delegaciones nacionales que asistan a las reuniones de trabajo acreditadas por cada organismo nacional de normalización, tienen garantizada su presencia representantes de las Àdministraciones de cada país con el fin de mantener la continuidad con la estructura que anteriormente tenía el tema de los Eurocódigos, cuando dependía directamente de la Comisión de la Comunidades Europeas. Así mismo, la publicación de un Eurocódigo como norma europea no llevará aparejado, como en los casos habituales, la anulación de las normas correspondientes que existan en cada país sobre la materia, sino que ambas podrán coexistir durante un período que se fijará por mutuo acuerdo entre la Comisión de la CE, el CEN y los paises miembros.

\section{OTROS ORGANISMOS EUROPEOS RELACIONADOS CON LA DIRECTIVA}

La Directiva recoge, además de las normas armonizadas, los Documentos de Idoneidad Técnica como especificaciones técnicas. Debemos indicar que estos documentos tienen dos aspectos diferenciales respecto a las normas, que están destinados a especificar las características de aquellos productos no tradicionales en la construcción, pues en caso contrario serian objeto de normas armonizadas y que tienen un carácter transitorio, pues permanecerán en tanto no haya normas para dichos productos.

Asi como todo el proceso de elaboración de las normas va a ser llevado a cabo por el CEN, las guias de los DIT europeos serán elaborados dentro de la Organización Europea de Acuerdos Técnicos (EOTA) que agrupa a las entidades que para estos documentos designe cada estado miembro. En el caso de España el organismo designado ha sido el Instituto de Ciencias de la Construcción Eduardo Torroja.

Dichas guias permitirán, sin perder la referencia a los documentos interpretativos, anteriormente comentados, conceder los DIT en base a ensayos, exámenes y evaluaciones para los productos de construcción no tradicionales.

Esta organización europea, la EOTA, debe complementarse con la antigua UEAtc, organismo que reunía a las entidades que en Europa venian concediendo y vigilando en cada pais la concesión de los DIT hasta la constitución de la EOTA.

La estrecha relación yła difusa frontera que en muchos casos separa si un producto es o no tradicional y por tanto si su especificación debe seguir el camino de la norma o del DIT, respectivamente, hizo necesario la firma de un acuerdo entre los dos organismos europeos que elaboraban normas y documentos de idoneidad, es decir, CEN y UEAtc. Ahora, y ante la reciente constitución de la EOTA, se ha vuelto a poner de manifiesto la necesidad de colaboración entre esta organización y el CEN. Las conversaciones ya se han iniciado y las primeras reuniones entre cualificados representantes de ambas entidades ya se han celebrado. Es de esperar que pronto cristalicen en un acuerdo que fije los campos de actuación de cada uno y los procedimientos de colaboración. Esta cooperación resulta necesaria para evitar posibles interferencias o solapes en sus trabajos y clarificar al industrial o fabricante europeo, el camino a seguir para especificar sus productos dentro del marco de la Directiva.

\section{LA NORMALIZACIÓN ESPAÑOLA EN CONSTRUCCIÓN}

Después de todo lo indicado, se comprende fácilmente que la actividad normalizadora a nivel nacional está centrada y condicionada por la actividad europea.

Centrada, porque no tendría sentido ignorar o no dedicar una atención preferencial a las normas que en los Comités europeos se están elaborando y que obligatoriamente van a ser, cuando se aprueben, adoptadas como normas nacionales UNE sustituyendo a lo que hasta ahora estuviera normalizado sobre el tema. 
Condicionada, porque los compromisos internacionales de "statu quo" nos impiden editar normas UNE en aquellas materias para las que se están elaborando normas europeas.

Con estas coordenadas se comprende que la actividad de los Comités Técnicos de Normalización en Construcción de AENOR tienen prácticamente completa su actividad con realizar el seguimiento de los respectivos Comités europeos de los que son responsables. En el anexo 2 se recoge la relación de Comités Técnicos de AENOR (AEN/CTN) que realizan el seguimiento de los trabajos de normalización que llevan a cabo los Comités Técnicos de-CEN (CEN/TC) del sector de construcción.

Debemos aprovechar esta oportunidad para hacer una, vez más, un llamamiento a la activa participación española en las reuniones internacionales; es alli donde se están elaborando las normas que pronto van a formar parte de nuestro cuerpo normativo, además van a ser las reglas técnicas del próximo mercado único europeo. Esta participación para que sea más efectiva debe ir precedida de un profundo análisis de los documentos a debate, y de una adopción de posturas en los Comités técnicos nacionales responsables.

Reconocemos que el esfuerzo es grande, sobre todo en el aspecto económico, pero hay que realizarlo ahora y no admite pausas pues el tren de la normalización europea lleva su velocidad, no se para, sino que se acelera si cabe.

\section{CONCLUSIÓN}

La situación que hemos descrito en el presente trabajo y la información en el contenido, demuestra que el comercio europeo de los productos de construcción va a venir regido en sus aspectos técnicos por unas reglas (normas armonizadas y DITES) que están elaborándose, luego las circunstancias exigen una urgente concienciación de la industria nacional para poder alcanzar un grado de desarrollo en estos temas equiparable al que tienen los otros paises miembros de la Comunidad Europea. En algunos subsectores ese grado de sensibilización ya ha alcanzado un nivel significativo, pero aún son bastantes los subsectores que deberían reaccionar prontamente para encarar en buena posición el inmediato futuro. 


\section{ANEXO 1}

\section{CEN/TC DEL SECTOR DE CONSTRUCCIÓN}

\begin{tabular}{|c|c|c|}
\hline CEN/TC & TÍTULO & TEMAS EN ESTUDIO \\
\hline 33 & $\begin{array}{l}\text { PUERTAS, VENTANAS, } \\
\text { PERSIANAS Y } \\
\text { HERRAJES. }\end{array}$ & $\begin{array}{l}\text { Clasificación, terminología, características y métodos de en- } \\
\text { sayo de puertas, ventanas, persianas y herrajes. Puertas in- } \\
\text { dustriales. Muros cortina. }\end{array}$ \\
\hline 50 & $\begin{array}{l}\text { CANDELABROS Y } \\
\text { SUS ACCESORIOS }\end{array}$ & $\begin{array}{l}\text { Definición, características y métodos de ensayo de los can- } \\
\text { delabros públicos de iluminación. Candelabros de acero, alea- } \\
\text { ciones de aluminio, hormigón armado y hormigón } \\
\text { pretensado... }\end{array}$ \\
\hline 51 & $\begin{array}{l}\text { CEMENTOS Y CALES } \\
\text { DE CONSTRUCCIÓN }\end{array}$ & $\begin{array}{l}\text { Definición, terminología, especificaciones y métodos de en- } \\
\text { sayo de los tipos de cementos y cales utilizados en la cons- } \\
\text { trucción de edificios e ingeniería civil. }\end{array}$ \\
\hline 53 & $\begin{array}{l}\text { ANDAMIOS. } \\
\text { PUNTALES Y } \\
\text { ANDAMIOS MÓVILES }\end{array}$ & $\begin{array}{l}\text { Proyecto, métodos de ensayo y requisitos de los andamios } \\
\text { y sus componentes. Andamios móviles, torres, puntales, re- } \\
\text { des de seguridad, puntales telescópicos... }\end{array}$ \\
\hline 67 & $\begin{array}{l}\text { BALDOSAS } \\
\text { CERÁMICAS }\end{array}$ & $\begin{array}{l}\text { Terminología, caracteristicas técnicas y métodos de ensayo } \\
\text { y control de las baldosas cerámicas, así como adhesivos es- } \\
\text { peciales para su colocación. }\end{array}$ \\
\hline 88 & $\begin{array}{l}\text { MATERIALES Y } \\
\text { PRODUCTOS } \\
\text { AISLANTES } \\
\text { TÉRMICOS }\end{array}$ & $\begin{array}{l}\text { Definiciones, terminología, propiedades, métodos de ensa- } \\
\text { yo de los materiales y productos de aislamiento térmico ta- } \\
\text { les como lana mineral, espuma de poliestireno expandido y } \\
\text { extruido, espuma de poliuretano y polisocianurato, espuma } \\
\text { fenólica, vidrio celular, lana de madera, bloques de perlita ex- } \\
\text { pandida, corcho, vermiculita... }\end{array}$ \\
\hline 89 & $\begin{array}{l}\text { EXIGENCIAS } \\
\text { TÉRMICAS DE } \\
\text { EDIFICIOS Y } \\
\text { SUS COMPONENTES }\end{array}$ & $\begin{array}{l}\text { Características térmicas de los edificios, y de sus compo- } \\
\text { nentes, puentes térmicos y superficie de condensación, } \\
\text { cálculo del consumo de energia, transmisión de calor a tra- } \\
\text { vés del suelo, propiedades térmicas de puertas y ventanas, } \\
\text { datos climatológicos. }\end{array}$ \\
\hline 99 & $\begin{array}{l}\text { REVESTIMIENTOS } \\
\text { DE PAREDES }\end{array}$ & $\begin{array}{l}\text { Revestimientos murales destinados a ser colocados con ad- } \\
\text { hesivos. Incluyendo los revestimientos murales acabados, los } \\
\text { destinados a una decoración posterior, los revestimientos tex- } \\
\text { tiles, de papel, plásticos y vinílicos. }\end{array}$ \\
\hline 104 & $\begin{array}{l}\text { HORMIGÓN. } \\
\text { PRODUCCIÓN, } \\
\text { PUESTA EN OBRA } \\
\text { Y CRITERIOS DE } \\
\text { CONFORMIDAD }\end{array}$ & $\begin{array}{l}\text { Propiedades del hormigón fresco y endurecido. Producción, } \\
\text { transporte, compactación y curado del hormigón fresco. Adi- } \\
\text { tivos, cenizas volantes, agua de amasado, pastas para hor- } \\
\text { migón postensado, vainas para armaduras pretensadas, pro- } \\
\text { tección y reparación de estructuras de hormigón. }\end{array}$ \\
\hline 112 & $\begin{array}{l}\text { TABLEROS A BASE } \\
\text { DE MADERA }\end{array}$ & $\begin{array}{l}\text { Terminologia, clasificación, requisitos y métodos de ensayo } \\
\text { de los tableros de partículas, contrachapados, tableros de fi- } \\
\text { bras, formaldehido,... }\end{array}$ \\
\hline 124 & $\begin{array}{l}\text { ESTRUCTURAS DE } \\
\text { MADERA }\end{array}$ & $\begin{array}{l}\text { Ejecución de estructuras de madera maciza, madera lamina- } \\
\text { da encolada y las uniones mecánicas. }\end{array}$ \\
\hline
\end{tabular}




\begin{tabular}{|c|c|c|}
\hline \multicolumn{3}{|l|}{$\ldots / \ldots$} \\
\hline 127 & $\begin{array}{l}\text { SEGURIDAD CONTRA } \\
\text { INCENDIOS EN } \\
\text { EDIFICIOS }\end{array}$ & $\begin{array}{l}\text { Determinación del comportamiento al fuego de los produc- } \\
\text { tos de construcción, así como de sus componentes y ele- } \\
\text { mentos. Clasificación de los productos de construcción se- } \\
\text { gún su utilización y los riesgos de incendio. }\end{array}$ \\
\hline 128 & $\begin{array}{l}\text { PRODUCTOS DE } \\
\text { COLOCACIÓN } \\
\text { DISCONTINUA PARA } \\
\text { CUBIERTAS }\end{array}$ & $\begin{array}{l}\text { Especificación y métodos de ensayo de tejas de hormigón, } \\
\text { tejas de arcilla, productos de fibrocemento. Placas bitumi- } \\
\text { nosas, chapas metálicas, pizarra y piedra natural. Placas } \\
\text { translúcidas, canalones y bajantes, así como los accesorios } \\
\text { utilizados en la construcción de cubiertas. }\end{array}$ \\
\hline 129 & $\begin{array}{l}\text { VIDRIO PARA } \\
\text { CONSTRUCCIÓN. }\end{array}$ & $\begin{array}{l}\text { Definición, clasificación, características, métodos de ensa- } \\
\text { yo y puesta en obra del vidrio utilizado en la construcción. } \\
\text { Vidrio laminado, templado y aislante, bloques de vidrio, vi- } \\
\text { drio de seguridad, vidrio para estructuras. }\end{array}$ \\
\hline 134 & $\begin{array}{l}\text { REVESTIMIENTOS } \\
\text { TÉXTILES Y } \\
\text { RESILIENTES } \\
\text { PARA SUELOS }\end{array}$ & $\begin{array}{l}\text { Especificación y métodos de ensayo de productos téxtiles, } \\
\text { caucho, plástico, corcho y linoleo para solados. }\end{array}$ \\
\hline 135 & $\begin{array}{l}\text { EJECUCIÓN DE } \\
\text { ESTRUCTURAS } \\
\text { DE ACERO }\end{array}$ & $\begin{array}{l}\text { Exigencias generales de fabricación y montaje de las estruc- } \\
\text { turas de acero. }\end{array}$ \\
\hline 154 & ÁRIDOS & $\begin{array}{l}\text { Especificaciones, toma de muestras y métodos de ensayo } \\
\text { de los áridos naturales y sintéticos utilizados para morteros } \\
\text { y hormigones. }\end{array}$ \\
\hline 163 & $\begin{array}{l}\text { APARATOS } \\
\text { SANITARIOS }\end{array}$ & $\begin{array}{l}\text { Especificaciones y métodos de ensayo de los aparatos sani- } \\
\text { tarios de cualquier material, inodoros, urinarios, lavabos, bi- } \\
\text { dés, fregaderos, bañeras, bañeras de hidromasaje, platos de } \\
\text { ducha y mamparas. }\end{array}$ \\
\hline 164 & $\begin{array}{l}\text { SUMINISTRO } \\
\text { DE AGUA }\end{array}$ & $\begin{array}{l}\text { Instalación y requisitos de funcionamiento de los sistemas } \\
\text { para suministro de agua, incluyendo el tratamiento de la mis- } \\
\text { ma. Tuberías de hormigón. Tuberías de fibrocemento, válvu- } \\
\text { las y accesorios, griferia, depósitos de agua fría y caliente... }\end{array}$ \\
\hline 165 & $\begin{array}{l}\text { INGENIERIA DE LAS } \\
\text { AGUAS RESIDUALES }\end{array}$ & $\begin{array}{l}\text { Instalación y requisitos de funcionamiento de los sistemas } \\
\text { de evacuación de aguas residuales, inćluyendo plantas de } \\
\text { tratamiento. Tuberias de gres, plástico, fibrocemento, fundi- } \\
\text { ción, acero y hormigón, tapas de registro, sifones, triturado- } \\
\text { res de basura, fosas asépticas... }\end{array}$ \\
\hline 166 & CHIMENEAS & $\begin{array}{l}\text { Conductos de humos destinados a la evacuación de los pro- } \\
\text { ductos de combustión desde la salida de los generadores } \\
\text { hasta la atmósfera. Conductos cerámicos, de hormigón y } \\
\text { metálicos. }\end{array}$ \\
\hline 167 & $\begin{array}{l}\text { APOYOS } \\
\text { ESTRUCTURALES }\end{array}$ & $\begin{array}{l}\text { Diseño, materiales, puesta en obra y métodos de ensayo de } \\
\text { los aparatos de apoyo utilizados en puentes, estadios, naves } \\
\text { industriales... apoyos elastoméricos, rodillos, rótulas, apoyos } \\
\text { "pot". }\end{array}$ \\
\hline
\end{tabular}




\begin{tabular}{|c|c|c|}
\hline \multicolumn{3}{|c|}{$\ldots / \ldots$} \\
\hline 177 & $\begin{array}{l}\text { COMPONENTES } \\
\text { PREFABRICADOS } \\
\text { ARMADOS DE } \\
\text { HORMIGÓN CURADO } \\
\text { EN AUTOCLAVE O } \\
\text { LIGERO SIN FINOS }\end{array}$ & $\begin{array}{l}\text { Especificación y métodos de ensayo de los elementos pre- } \\
\text { fabricados de hormigón utilizados en muros, tejados, } \\
\text { forjados... }\end{array}$ \\
\hline 125 & ALBAÑ̃LERÍA & $\begin{array}{l}\text { Especificaciones y métodos de ensayo de bloques de: cerá- } \\
\text { mica, silicato cálcico, hormigón, piedra natural y artificial. } \\
\text { Morteros, anclajes, dinteles y armaduras para juntas utiliza- } \\
\text { das en albañilería. }\end{array}$ \\
\hline 126 & $\begin{array}{l}\text { PROPIEDADES } \\
\text { ACÚSTICAS DE LOS } \\
\text { EDIFICIOS Y SUS } \\
\text { ELEMENTOS } \\
\text { CONSTRUCTIVOS }\end{array}$ & $\begin{array}{l}\text { Métodos de medición en laboratorio e "IN SITU". Transmi- } \\
\text { - sión de las propiedades de los productos a las propiedades } \\
\text { del edificio. }\end{array}$ \\
\hline 178 & $\begin{array}{l}\text { UNIDADES PARA } \\
\text { PAVIMENTOS Y } \\
\text { BORDILLOS }\end{array}$ & $\begin{array}{l}\text { Especificación y métodos de ensayo de losas para pavimen- } \\
\text { tos y bordillos prefabricados de hormigón, de piedra natural } \\
\text { o cerámicos. }\end{array}$ \\
\hline 189 & $\begin{array}{l}\text { GEOTEXTILES Y } \\
\text { PRODUCTOS } \\
\text { RELACIONADOS }\end{array}$ & $\begin{array}{l}\text { Terminología, clasificación y métodos de ensayo de los } \\
\text { geotextiles. }\end{array}$ \\
\hline 217 & $\begin{array}{l}\text { SUPERFICIES } \\
\text { DEPORTIVAS }\end{array}$ & $\begin{array}{l}\text { Especificación y métodos de ensayo de las superficies para } \\
\text { salas y pabellones deportivos, césped natural y artificial, su- } \\
\text { perficies minerales aglomeradas, superficies sintéticas. }\end{array}$ \\
\hline 227 & $\begin{array}{l}\text { CONSTRUCCIÓN Y } \\
\text { MANTENIMIENTO } \\
\text { DE CARRETERAS }\end{array}$ & $\begin{array}{l}\text { Especificación, métodos de ensayo en la construcción de ca- } \\
\text { rreteras. Sus materiales de mantenimiento. Mezclas bitumi- } \\
\text { nosas, revestimientos superficiales, materiales para carrete- } \\
\text { ras de hormigón... }\end{array}$ \\
\hline 229 & $\begin{array}{l}\text { PRODUCTOS } \\
\text { PREFABRICADOS } \\
\text { DE HORMIGÓN }\end{array}$ & $\begin{array}{l}\text { Especificaciones y métodos de ensayo de los productos pre- } \\
\text { fabricados de hormigón. Peldaños, barandillas, recubrimien- } \\
\text { tos para suelos (usos agricolas), losas alveoladas, postes, } \\
\text { terrazo... }\end{array}$ \\
\hline 241 & $\begin{array}{l}\text { YESO Y PRODUCTOS } \\
\text { DE YESO }\end{array}$ & $\begin{array}{l}\text { Especificaciones y métodos de ensayo del yeso en polvo, pla- } \\
\text { cas a base de sulfato cálcico, bloques de yeso,... Así como } \\
\text { de los accesorios utilizados para su colocación. }\end{array}$ \\
\hline 246 & ROCAS NATURALES & $\begin{array}{l}\text { Terminologia, clasificación, características y métodos de en- } \\
\text { sayo de las rocas naturales ornamentales. }\end{array}$ \\
\hline 247 & $\begin{array}{l}\text { DISPOSITIVOS DE } \\
\text { CONTROL DE } \\
\text { LOS SERVICIOS } \\
\text { MECÁNICOS DE } \\
\text { LOS EDIFICIOS }\end{array}$ & $\begin{array}{l}\text { Sistemas y dispositivos destinados al control automático y } \\
\text { manual de las instalaciones de los edificios, en particular los } \\
\text { sistemas de calefacción y ventilación. }\end{array}$ \\
\hline $250^{\circ}$ & $\begin{array}{l}\text { EUROCÓDIGOS } \\
\text { ESTRUCTURALES }\end{array}$ & $\begin{array}{l}\text { Bases para el diseño y cálculo de las estructuras de hormi- } \\
\text { gón, de acero, mixtas, de madera y de albañilería para edifi- } \\
\text { cios e ingeniería civil. }\end{array}$ \\
\hline 254 & $\begin{array}{l}\text { LÁMINAS FLEXIBLES } \\
\text { PARA } \\
\text { IMPERMEABILIZACIÓN }\end{array}$ & $\begin{array}{l}\text { Especificaciones y métodos de ensayo de las láminas bitu- } \\
\text { minosas y sintéticas utilizadas en impermeabilización. }\end{array}$ \\
\hline
\end{tabular}


CORRESPONDENCIA ENTRE AEN/CTN Y CEN/TC DEL ÁREA DE CONSTRUCCIÓN

\begin{tabular}{|c|c|c|}
\hline AEN/CTN & TÍTULO & CEN/TC RELACIONADOS \\
\hline 19 & $\begin{array}{l}\text { "TUBERIAS DE FUNDICIÓN, } \\
\text { GRIFERÍA, VALVULERIA Y } \\
\text { ACCESORIOS DE MATERIALES } \\
\text { METÁLICOS" }\end{array}$ & $\begin{array}{l}164 \text { "SUMINISTRO DE AGUA" } \\
165 \text { "INGENIERÍA DE LAS AGUAS RESIDUALES" }\end{array}$ \\
\hline 22 & "MINERÍA Y EXPLOSÍVOS" & $\begin{array}{l}125 \text { "ALBAÑILERIA" } \\
128 \text { "PRODUCTOS DE COLOCACIÓN DISCONTINUA } \\
\text { PARA CUBIERTAS" } \\
178 \text { "UNIDADES PARA PAVIEMENTOS Y BORDILLOS" } \\
246 \text { "PIEDRA NATURAL" }\end{array}$ \\
\hline 23 & $\begin{array}{l}\text { "SEGURIDAD CONTRA } \\
\text { INCENDIOS" }\end{array}$ & $\begin{array}{l}127 \text { "SEGURIDAD CONTRA INCENDIOS EN EDIFI- } \\
\text { CIOS" }\end{array}$ \\
\hline 40 & "INDUSTRIAS TÉXTILES" & $\begin{array}{l}134 \text { "REVESTIMENTOS TÉXTILES Y RESILIENTES PA- } \\
\text { RA SUELOS" } \\
189 \text { "GEOTEXTILES Y PRODUCTOS RELACIONADOS" }\end{array}$ \\
\hline 41 & "CONSTRUCCIÓN" & $\begin{array}{l}125 \text { "ALBAÑILERÍA" } \\
128 \text { "PRODUCTOS DE COLOCACIÓN DISCONTINUA } \\
\text { PARA CUBIERTAS" } \\
164 \text { "SUMINISTRO DE AGUA" } \\
165 \text { "INGENIERÍA DE LAS AGUAS RESIDUALES" } \\
217 \text { "SUPERFICIES DEPORTIVAS" } \\
227 \text { "CONSTRUCCIÓN Y MANTENIMIENTO DE CA- } \\
\text { RRETERAS" }\end{array}$ \\
\hline 43 & "INDUSTRIAS DEL VIDRIO" & 129 "VIDRIO PARA CONSTRUCCIÓN" \\
\hline 53 & "PLÁSTICOS Y CAUCHO" & $\begin{array}{l}128 \text { "PRODUCTOS DE COLOCACIÓN DISCONTINUA } \\
\text { PARA CUBIERTAS” } \\
134 \text { "REVESTIMIENTOS TEXTILES Y RESILIENTES } \\
\text { PARA SUELOS” } \\
163 \text { "APARATOS SANITARIOS" } \\
165 \text { "INGENIERIA DE LAS AGUAS RESIDUALES" } \\
167 \text { "APOYOS ESTRUCTURALES" }\end{array}$ \\
\hline 56 & "MADERA Y CORCHO" & $\begin{array}{l}33 \text { “PUERTAS, VENTANAS, PERSIANAS Y HERRA- } \\
\text { JES" } \\
112 \text { "TABLEROS A BASE DE MADERA" } \\
124 \text { "ESTRUCTURAS DE MADERA" } \\
134 \text { "REVESTIMIENTOS TEXTILES Y RESILIENTES } \\
\text { PARA SUELOS" }\end{array}$ \\
\hline 57 & "CELULOSA Y PAPEL" & 99. "REVESTIMIENTOS DE PAREDES" \\
\hline 67 & $\begin{array}{l}\text { "PRODUCTOS CERÁMICOS } \\
\text { PARA LA CONSTRUCCIÓN" }\end{array}$ & 163 "APARATOS SANITARIOS” \\
\hline 72 & "ILUMINACIÓN Y COLOR" & 50 "CANDELABROS Y SUS ACCESORIOS" \\
\hline 74 & "ACÚSTICA" & $\begin{array}{l}126 \text { "PROPIEDADES ACÚSTICAS DE LOS EDIFICIOS } \\
\text { Y SUS ELEMENTOS CONSTRUCTIVOS" } \\
129 \text { "VIDRIO PARA CONSTRUCCIÓN" }\end{array}$ \\
\hline
\end{tabular}




\begin{tabular}{|c|c|c|}
\hline 76 & “ESTRUCTURAS METÁLICAS" & $\begin{array}{l}53 \text { "ANDAMIOS. PUNTALES Y ANDAMIOS MÓVILES" } \\
135 \text { "EJECUCIÓN DE ESTRUCTURAS DE ACERO" } \\
167 \text { "APOYOS ESTRUCTURALES" }\end{array}$ \\
\hline 80 & "CEMENTOS Y CALES" & 51 "CEMENTOS Y CALES DE CONSTRUCCIÓN" \\
\hline 83 & "HORMIGÓN" & $\begin{array}{l}104 \text { "HORMIGÓN. PRODUCCIÓN, PUESTA EN OBRA } \\
\text { Y CRITERIOS DE CONFORMIDAD" } \\
125 \text { "ALBAÑILERIA" } \\
154 \text { "ÁRIDOS" }\end{array}$ \\
\hline 85 & $\begin{array}{l}\text { "PUERTAS, VENTANAS, } \\
\text { PERSIANAS, HERRAJES Y, } \\
\text { CERRADURAS" }\end{array}$ & $\begin{array}{l}33 \text { "PUERTAS, VENTANAS, PERSIANAS Y HERRA- } \\
\text { JES" }\end{array}$ \\
\hline 88 & $\begin{array}{l}\text { "PRODUCTOS DE CEMENTO } \\
\text { REFORZADO CON FIBRAS" }\end{array}$ & $\begin{array}{l}128 \text { "PRODUCTOS DE COLOCACIÓN DISCONTINUA } \\
\text { PARA CUBIERTAS" } \\
164 \text { "SUMINISTRO DE AGUA" } \\
165 \text { "INGENIERIAA DE LAS AGUAS RESIDUALES" }\end{array}$ \\
\hline 92 & "AISLAMIENTO TÉRMICO" & $\begin{array}{l}88 \text { "MATERIALES Y PRODUCTOS AISLANTES TÉR- } \\
\text { MICOS" }\end{array}$ \\
\hline 100 & “CLIMATIZACIÓN" & $\begin{array}{l}89 \text { "EXIGENCIAS TÉRMICAS DE EDIFICIOS Y SUS } \\
\text { COMPONENTES” } \\
247 \text { "DISPOSITIVOS DE CONTROL DE LOS SERVI- } \\
\text { CIOS MECÁNICOS DE LOS EDIFICIOS" }\end{array}$ \\
\hline 102 & $\begin{array}{l}\text { "YESO Y PRODUCTOS A BASE } \\
\text { DE YESO" }\end{array}$ & 241 "YESO Y PRODUCTOS DE YESO" \\
\hline 104 & $\begin{array}{l}\text { "MATERIALES } \\
\text { IMPERMEABILIZANTES PARA } \\
\text { LA CONSTRUCCIÓN" }\end{array}$ & $\begin{array}{l}128 \text { "PRODUCTOS DE COLOCACIÓN DISCONTINUA } \\
\text { PARA CUBIERTAS" } \\
254 \text { "LÁMINAS FLEXIBLES PARA IMPERMEABILIZA- } \\
\text { CIÓN" }\end{array}$ \\
\hline 123 & "CHIMENEAS" & 166 "CHIMENEAS" \\
\hline 127 & $\begin{array}{l}\text { "PREFABRICADOS DE } \\
\text { CEMENTO Y HORMIGÓN" }\end{array}$ & $\begin{array}{l}125 \text { "ALBAÑILERÍA" } \\
128 \text { "PRODUCTOS DE COLOCACIÓN DISCONTINUA } \\
\text { PARA CUBIERTAS" } \\
165 \text { "INGENIERÍA DE LAS AGUAS RESIDUALES" } \\
177 \text { "COMPONENTES PREFABRICADOS ARMADOS } \\
\text { DE HORMIGÓN CURADO EN AUTOCLAVE O LIGE- } \\
\text { RO SIN FINOS" } \\
178 \text { "UNIDADES PARA PAVIMENTOS Y BORDILLOS" } \\
229 \text { "PRODUCTOS PREFABRICADOS DE HORMIGÓN" }\end{array}$ \\
\hline 136 & $\begin{array}{l}\text { "MATERIALES CERÁMICOS } \\
\text { DE ARCILLA COCIDA PARA } \\
\text { LA CONSTRUCCIÓN" }\end{array}$ & $\begin{array}{l}125 \text { "ALBAÑILERÍA" } \\
128 \text { "PRODUCTOS DE COLOCACIÓN DISCONTINUA } \\
\text { PARA CUBIERTAS" } \\
178 \text { "UNIDADES PARA PAVIMENTOS Y BORDILLOS" }\end{array}$ \\
\hline 138 & "BALDOSAS CERÁMICAS" & 67 “BALDOSAS CERÁMICAS" \\
\hline 140 & $\begin{array}{l}\text { "EUROCÓDIGOS } \\
\text { ESTRUCTURALES" }\end{array}$ & 250 "EUROCÓDIGOS ESTRUCTURALES" \\
\hline
\end{tabular}

